\title{
Immobilization of chitosan gel with cross-linking reagent on PNIPAAm gel/PP nonwoven composites surface
}

\author{
Ko-Shao Chen ${ }^{\mathrm{a}, *}$, Yuan-An Ku ${ }^{\mathrm{a}}$, Chi-Han Lee ${ }^{\mathrm{a}}$, Hong-Ru Lin ${ }^{\mathrm{b}}$, Feng-Huei Lin ${ }^{\mathrm{c}}$, Tim-Mo Chen ${ }^{\mathrm{d}}$ \\ ${ }^{a}$ Department of Materials Engineering, Tatung University, 40 Chungshan N. RD. 3rd Sec., Taipei 10451, Taiwan \\ ${ }^{\mathrm{b}}$ Department of Chemical Engineering, Southern Taiwan University of Technology, Tainan, Taiwan \\ ${ }^{\mathrm{c}}$ Institute of Biomedical Engineering, College of Engineering and Medicine, National Taiwan University, Taipei, Taiwan \\ ${ }^{\mathrm{d}}$ Burn Center-Department of Surgery, Tri-service General Hospital, Taipei, Taiwan
}

Received 14 May 2004; received in revised form 1 November 2004; accepted 18 December 2004

Available online 26 February 2005

\begin{abstract}
This study was to immobilize chitosan (CS), which is a biodegradable and antibacterial polymer, on poly $(N$-isopropylacrylamide) (PNIPAAm) gel/polypropylene (PP) nonwoven composites surface for wound dressing applications. PP nonwoven has been extensively used due to its porosity, allowing ventilation, high surface area and excellent mechanical properties. However, the hydrophobic surface of PP nonwoven limits its applications; in this study, we used the plasma-activation treatment and subsequently UV-light graft polymerization of NIPAAm gel to improve its hydrophilicity. Chitosan was immobilized onto PNIPAAm gel/PP nonwoven composites surface using the cross-linking agent, glutaraldehyde (GA). This complex was characterized by scanning electron microscopy (SEM). The results indicated that the wettability of the composite was improved after plasma treatment and photo-induced graft polymerization and chitosan was successfully immobilized onto the surface of PNIPAAm gel/PP nonwoven composites through cross-linking process. Finally, the preliminary result shows that chitosan hydrogels displayed antibacterial ability to Escherichia coli and Staphylococcus aureus. The (3-(4, 5-dimethylthiazolyl-2)-2, 5-diphenyltetrazolium bromide) (MTT) method indicated that the porous chitosan sponge exhibited good biocompatibility to fibroblast cells.
\end{abstract}

(C) 2005 Elsevier B.V. All rights reserved.

Keywords: Chitosan; Poly(N-isopropylacrylamide); PP nonwoven; Plasma; Biocompatibility

\section{Introduction}

Skin defect of large areas caused by burns or injury is a firmly dangerous situation. Without immediate and adequate curing, patients might die or lost some mobile function of limbs [1]. In the clinic treatment, the instantly coverage of wound dressing or artificial skin to protect the wound is necessary [2-4]. During the last decade, many new wound dressings have been developed and marketed for wound management $[5,6]$. Silicon film

\footnotetext{
* Correspodning author. Tel.: +88622586 7150; fax: +886225936897.

E-mail addresses: kschen@ttu.edu.tw (K.-S. Chen), yuanan@ms36.url.com.tw (Y.-A. Ku).
}

combined with collagen layer is usually used for these products $[1,7,8]$. However, silicone layer limits the ventilation of body fluid and the collagen sponge material is very expensive. In order to help lightening the pain of patients suffered by changing dressing frequently, an easy stripped off function of the dressing should be introduced. Hydrophilic $N$-isopropylacrylamide (NIPAAm) monomer is a good material that has the easy stripped off function due to its thermo-sensitive critical property [9-11]. Poly( $N$-isopropylacrylamide) (PNIPAAm) exhibits a lower critical solution temperature (LCST) and remarkable hydration-dehydration changes in response to relatively small changes in temperature [12]. Below the LCST, around $32{ }^{\circ} \mathrm{C}$, PNIPAAm chains hydrate to form an expanded structure; above the LCST, PNIPAAm chains 

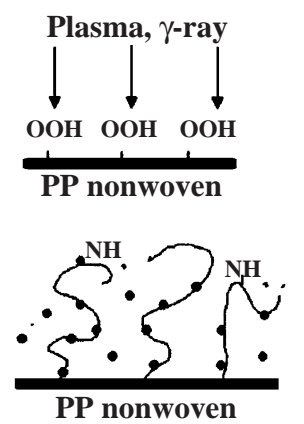

Fig. 1. Schematic diagram for the chemical reaction of photo-induced grafting polymerization.

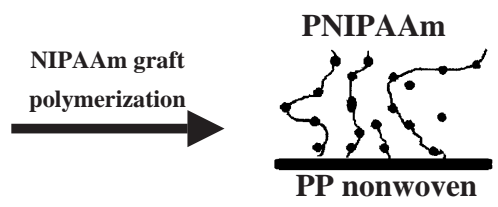

Chitosan gel

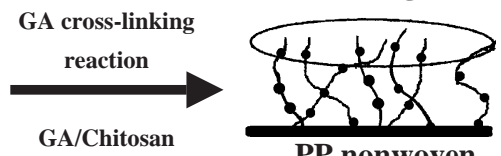

PP nonwoven dehydrate to form a shrinkage structure. Therefore, one can graft NIPAAm onto the surface of substrate, and when it needs removed, just let the temperature of the substrate below the LCST to make PNIPAAm swelling and then it would be easily stripped-off from the wound surface without damaging the new regenerated tissues [13-16].

Polysaccharides, e.g. chitosan, having hydrogel-forming properties have been considered to be advantageous in their application as a wound dressing materials [17-19]. Chitosan is partially $N$-deacetylated chitin, and chitin is a linear homopolymer of 1,4 $\beta$-linked $N$-acetyl-D-glucosamine. Both chitin and chitosan have many useful and advantageous biological properties in the application as a wound dressing, namely biocompatibility, biodegradability, hemostatic activity, anti-infectional activity, and property to accelerate wound healing [19-21].

In this study, porous nonwoven was adopted to replace the silicon film. The introduction of NIPAAm monomer onto PP nonwoven was performed and chitosan was immobilized onto PNIPAAm gel/nonwoven composites surface using the cross-linking reagent, glutaraldehyde (GA), which was bonded to chitosan by reaction between aldehyde group of the cross-linking reagent and a free amino group of chitosan. This complex was characterized by scanning electron microscopy (SEM). Antibacterial
Table 1

Effect of cross-linking conditions on characteristics of chitosan hydrogel

\begin{tabular}{|c|c|c|c|c|}
\hline \multicolumn{2}{|c|}{ Solution $(100 \mathrm{ml})$} & \multirow{2}{*}{$\begin{array}{l}\text { GA/Chitosan } \\
\text { ration }\end{array}$} & \multirow{2}{*}{$\begin{array}{l}\text { Mechanic } \\
\text { properties }\end{array}$} & \multirow[t]{2}{*}{ Hydrogel color } \\
\hline $\begin{array}{l}\text { Chitosan } \\
\text { (wt\%) }\end{array}$ & $\begin{array}{l}\text { GA } \\
(w t \%)\end{array}$ & & & \\
\hline 0.4 & 0.8 & 2 & Brittle & Dark-orange \\
\hline 0.5 & 0.75 & 1.5 & & \\
\hline 0.66 & 0.66 & 1 & & \\
\hline 1 & 0.5 & 0.5 & & \\
\hline 1.32 & 0.33 & 0.25 & & \\
\hline 1.5 & 0.25 & 0.17 & & \\
\hline 1.6 & 0.2 & 0.13 & Elasticity & Light-yellow \\
\hline 2 & 0 & 0 & - & Light-yellow \\
\hline
\end{tabular}

activities and biocompatibility of these complexes were investigated.

\section{Materials and method}

\subsection{Materials}

Polypropylene nonwoven was supplied by Chang Gung University (Tao-Yuan, Taiwan). The nonwoven was cut into strips $\left(2 \times 3 \mathrm{~cm}^{2}\right)$ and washed by $95 \%$ alcohol overnight to remove the residual substance. $N$-isopropylacrylamide (NIPAAm) was supplied by Eastman Kodack USA, Ammonium persulfate (APS, Wako Pure Chemical Industry), $N, N, N^{\prime}, N^{\prime}$-tetra-methylethylene-diamine (TEMED, Fluka), and $N, N^{\prime}$-methylene-bis-acrylamide (NMBA, Sigma). Chitosan powder was obtained from Prof. J.M. Yang (Chang Gung University, Tao-Yuan, Taiwan). Glutaradehyde (GA) was purchased from SHIYAKU with $25 \%$ extra pure concentration. Other agents or drugs were all chemical grade.

\subsection{Plasma treatment}

PP nonwoven was individually placed inside a bell-jar reactor with parallel electrode. The plasma activation

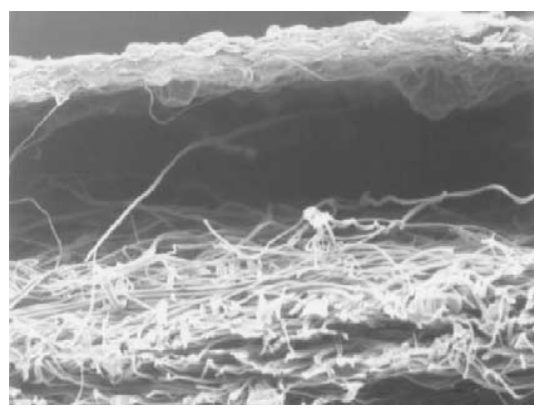

(a) $50 \mathrm{x}$

$1 \mathrm{~mm}$

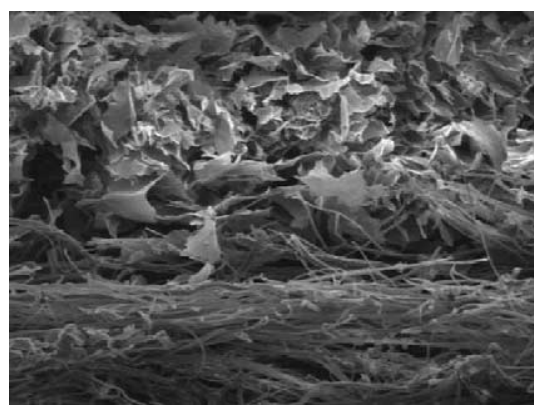

(b) $50 \mathrm{x}$
$1 \mathrm{~mm}$

Fig. 2. SEM of the PNIPAAm hydrogel on (a) untreated PP nonwoven and (b) plasma treated PP nonwoven. (Ar plasma: $150 \mathrm{mTorr}, 50 \mathrm{~W}, 10 \mathrm{~min}$; Graft: 1000 $\mathrm{W}, 15 \mathrm{~min}$.). 


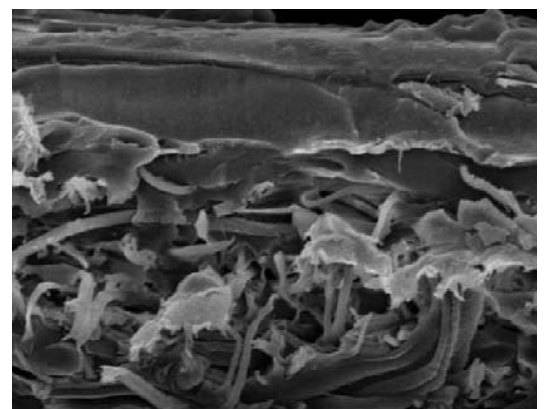

(a) $50 \mathrm{x}$
$1 \mathrm{~mm}$

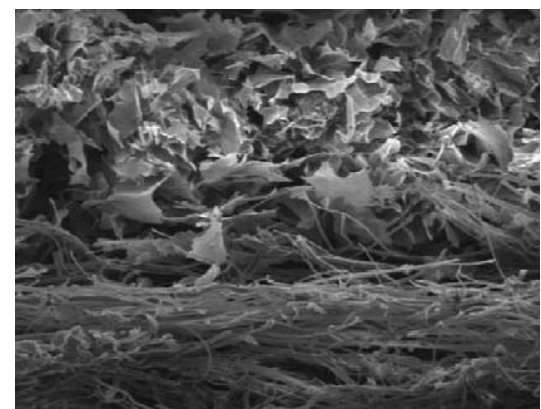

(b) $50 \mathrm{x}$
$1 \mathrm{~mm}$

Fig. 3. SEM of chitosan/PP nonwoven composites. (a) dried at room temperature and (b) freeze dried at $-80{ }^{\circ} \mathrm{C}$.

treatment system (SAMCO PD-2S model) equips with a parallel electrode in the reaction chamber. Prior to initiation of plasma state, the pressure in the chamber was pumped down to $50 \mathrm{mTorr}$ by vacuum pump. The pure argon gas was then introduced into the chamber and adjusting the micro-throttle valve until approximately 150 mTorr of pressure was reached. After the pressure was stable around 150 mTorr, the plasma state was activated for $10 \mathrm{~min}$ by RF power generator $(13.56 \mathrm{MHz})$ at $50 \mathrm{~W}$ [22-24].

\subsection{Photo-induced graft copolymerization}

Photo-induced graft polymerization on the substrate was carried out in a Pyrex glass container with $20 \mathrm{ml}$ aqueous NIPAAm solution (6.35 wt.\%), in which the given concentrations of additives [APS (0.11 wt. $\%)$, TEMED (0.14 wt.\%), NMBA (0.46 wt.\%)] were dissolved. Then $0.01 \mathrm{~g} / \mathrm{l}$ riboflavin $\left(\mathrm{B}_{2}, \mathrm{C}_{17} \mathrm{H}_{20} \mathrm{~N}_{4} \mathrm{O}_{6}\right.$, Sigma Chem.) was added in a ratio of NIPAAm solution/ riboflavin $=4: 1$. After the solutions was mixed completely and PP nonwoven was added, the Pyrex glass container was placed in a UV-light (wavelength: $365 \mathrm{~nm}$, Henchman, Taiwan) irradiated system with water-cooling devise. The irradiation reaction was carried out at room temperature by high-pressure mercury lamp (1000 W) [22-25]. After the reaction was complete, the substrate was took out and washed for $24 \mathrm{~h}$ with distilled water to remove the homo-polymer. The schematic diagram for the modification is shown in Fig. 1.

\subsection{Preparation of chitosan hydrogel}

$2 \mathrm{~g}$ of chitosan was stirred in $100 \mathrm{ml}$ of $0.1 \mathrm{M}$ acetic acid at room temperature until homogenous solution was obtained. Due to the $-\mathrm{NH}_{2}$ group of amino acid, chitosan can cross-link with cross-linking agent such as glutaraldehyde. The different methods of cross-link process are operated as below.

\subsubsection{One-step process}

Different concentrations of glutaraldehyde aqueous solutions were mixed with chitosan solution, respectively.
The mixture was instantly poured onto the PP nonwoven- $g$ PNIPAAm composite which was steady placed on the surface of dish. The cross-linking reaction was carried out at room temperature for a few hours. The hydrogels were treated with $0.1 \mathrm{M}$ glycine aqueous solution to block nonreacted aldehyde groups, and then washed thrice with double-distilled water.

\subsubsection{Two-step process}

The chitosan solution was poured onto the PP nonwoven-g-PNIPAAm hydrogel composite. Then, the specimen was put into the $-80{ }^{\circ} \mathrm{C}$ refrigerator for three hours and further placed into a freeze-dryer for 1-2 days to dry the specimen completely. Finally, this dry specimen was immersed into the glutaraldehyde solution to process the cross-link reaction overnight. After that, the cross-linked chitosan hydrogel was further treated with $0.1 \mathrm{M}$ glycine aqueous solution to block non-reacted aldehyde groups, and then washed thrice with double-distilled water.

\subsection{Preparaing of chitosan sponge}

The specimen was first placed in a $-80{ }^{\circ} \mathrm{C}$ refrigerator for about 3 hours and further put it into the freeze-dryer to dehydration for 1-2 days. A porous and sponge structure was prepared by this treatment.

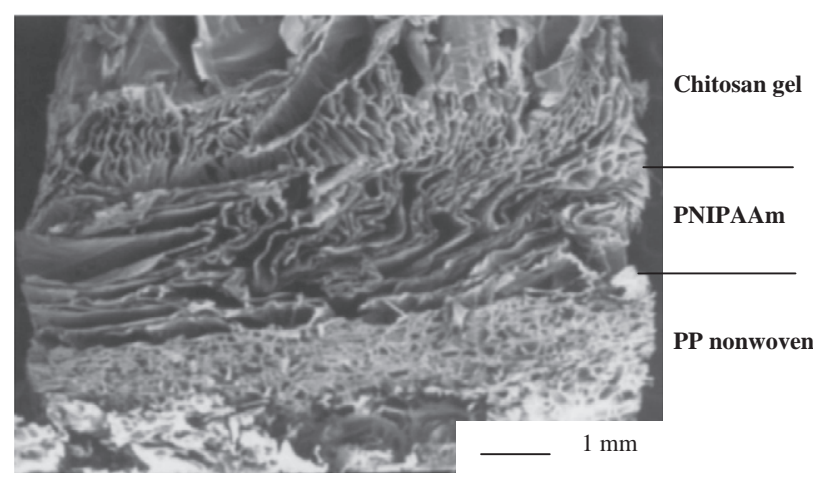

Fig. 4. SEM of tri-layer wound dressing structure by freeze dried at $-80^{\circ} \mathrm{C}$. $(50 \times)$. 


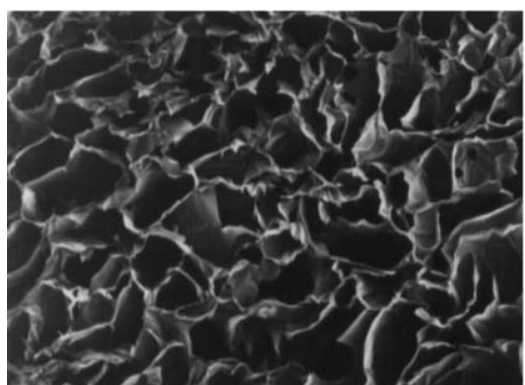

(a)

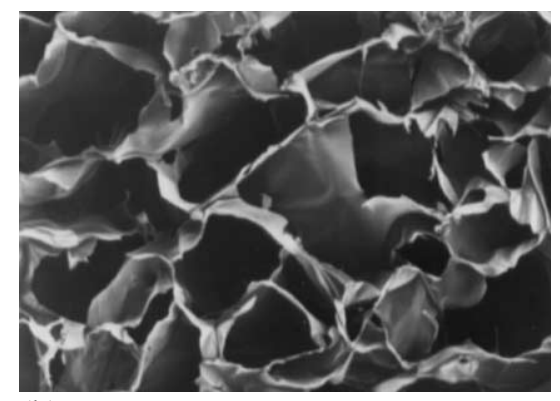

(b)
$100 \mu \mathrm{m}$

Fig. 5. SEM of the porous structures of freeze dried chitosan sponge layer. (a) magnification $50 \times$ and (b) magnification $100 \times$. (freeze dried at $-80{ }^{\circ} \mathrm{C}$ ).

\subsection{Characterization}

\subsubsection{SEM morphological observation}

Morphology has been playing an important role to determine the surface structure of nonwoven. For different treated nonwoven, the morphology was observed by scanning electron microscopy (Hitachi JSM-6300 model).

\subsubsection{Cell culture}

Fibroblasts were isolated from the human skin according to the conventional method by collagenase. Culture was maintained in a $37{ }^{\circ} \mathrm{C}$ water-jacketed incubator equilibrated with $5 \% \quad \mathrm{CO}_{2}$ and kept at approximately $99 \%$ relative humidity. The cells were routinely grown in RPMI-1640 medium containing 10\% fetal bovine serum (FBS; Gibco, USA) and 1\% antibiotic-antimycotic (Gibco, USA) in a $75 \mathrm{~cm}^{2}$ cell culture flask (Costar, USA). The cells were subcultured for about 3-day intervals with trypsin-EDTA (Gibco, USA) and used within seven to eight cycles of subculture after the primary culture.
The prepared specimens with $15 \mathrm{~mm}$ in diameter, sterilized in $70 \%$ alcohol overnight and rinsed extensively with distilled water, were placed in 24-welled tissue culture polystyrene plates (Costar, USA). A sterilized Teflon ring $15-\mathrm{mm}$ diameter was placed on each of the tested membranes in the wells to prevent them from floating. Then the specimens were washed twice with Dulbecco's PBS. After aspiration of PBS, 1-ml medium of cell suspension at a density of $2 \times 10^{5}$ cells/ml RPMI-1640 medium was placed on each well and maintained in a humidified atmosphere with $5 \% \mathrm{CO}_{2}$ at $37{ }^{\circ} \mathrm{C}$.

\subsubsection{MTT assay}

MTT reagent is a pale yellow substrate which produces a dark blue formazan product when it is incubated with viable cells. After cell culturing for 1,3 , and 5 days, the viability of fibroblasts was determined by MTT assay. The method of Mosmann [26] was modified and used in this study. At each period of culturing time, the 100 il MTT (Sigma; USA) solution was added to each well. After $4 \mathrm{~h}$ incubation at $37^{\circ} \mathrm{C}$,

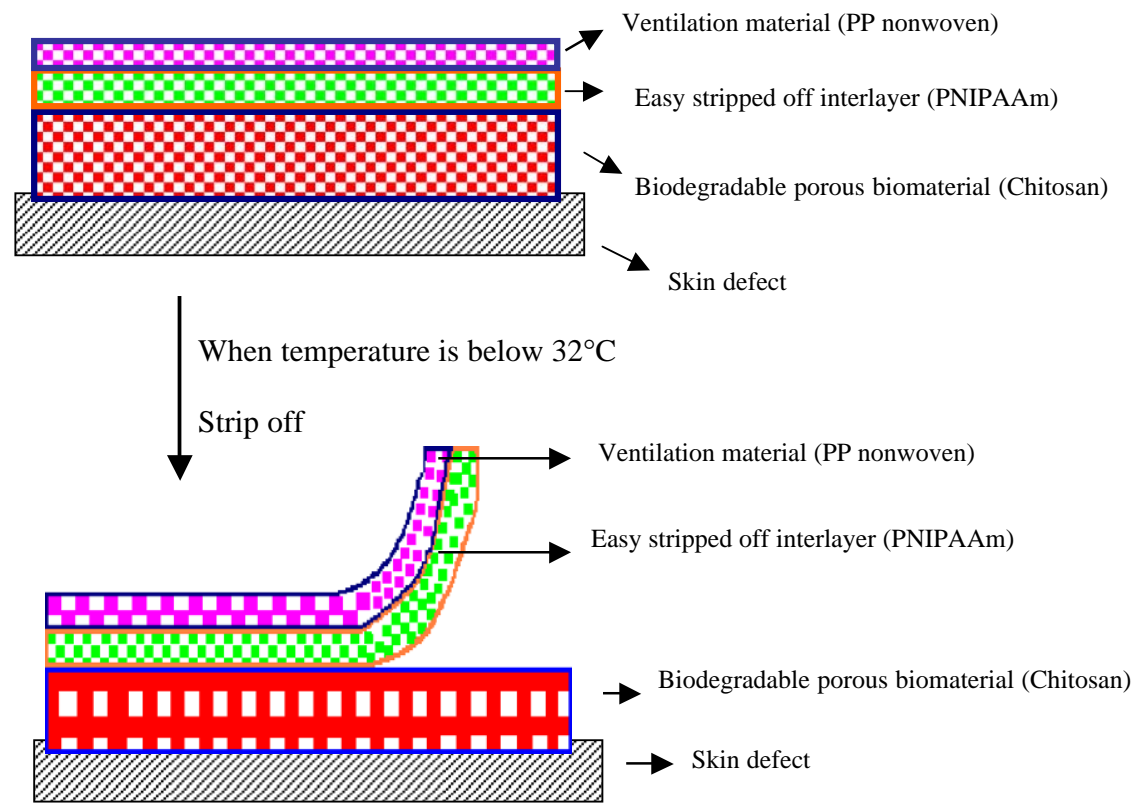

Fig. 6. The architecture of the easily stripped-off trilayer wound dressing. 
$200 \mu \mathrm{l}$ of DMSO (dimethyl sulfoxide) was added to dissolve the formazan crystals. The dissolvable solution was jogged homogeneously about $15 \mathrm{~min}$ by the shaker. The optical density of the formazan solution was read on an UV-VIS spectrophotometer (HMADZU UV-1201 model) at $570 \mathrm{~nm}$. All experiments were repeated four times.

\subsubsection{Assay for antibacterial activity}

Each hydrogel (2-8 mg) was washed with PBS for 30 min twice, sterilized in $70 \%$ ethanol for $30 \mathrm{~min}$ and then

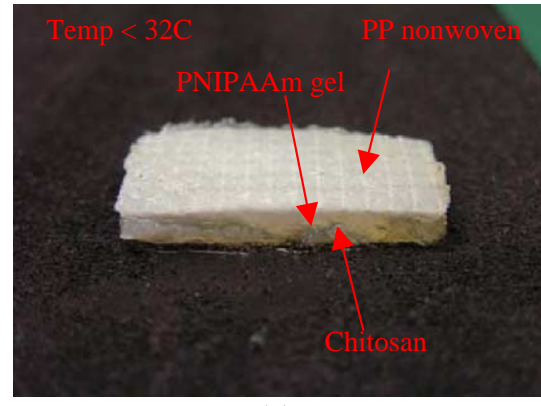

(a)

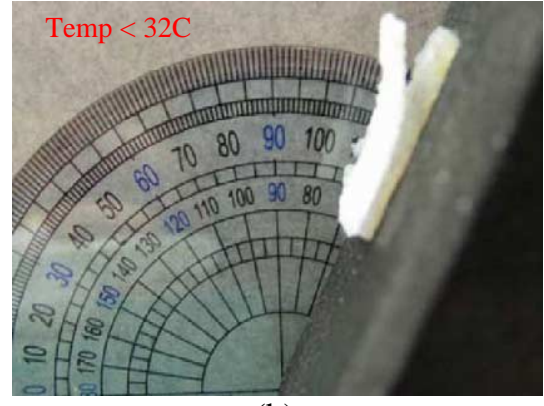

(b)

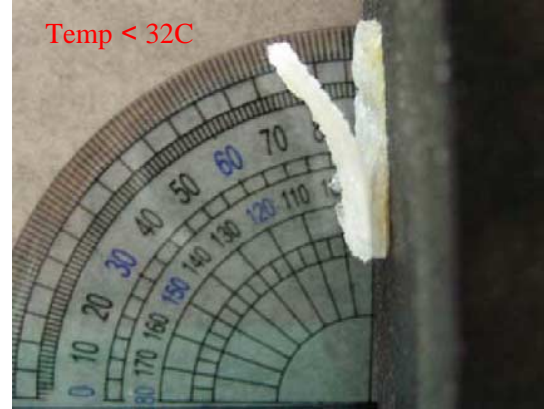

(c)

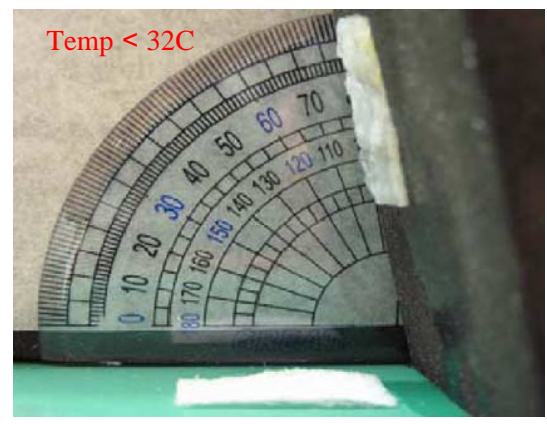

(d) dried in the clean cabinet. Fresh overnight culture of Escherichia coli and Staphylococcus aureus were diluted to 1000 or 10000 colony forming units (c.f.u.)/ml. The hydrogels were immersed separately in thus prepared $E$. coli and $S$. aureus suspension $(200 \mathrm{ml})$. After $2 \mathrm{~h}$ incubation at room temperature, one aliquot of bacteria suspension was spread onto LB plate to determine the bacteria number. The reduction rate in bacteria number was calculated by dividing the bacteria number of the hydrogel-treated sample by that of non-treated one.

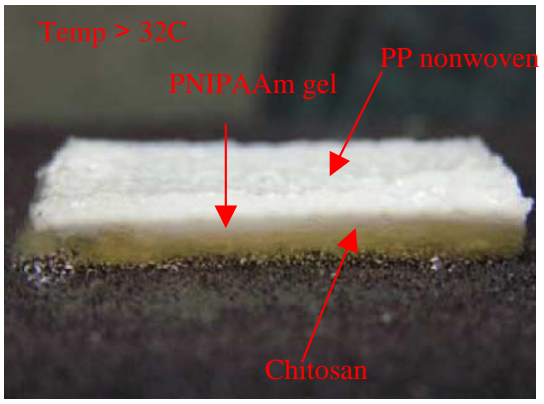

(e)

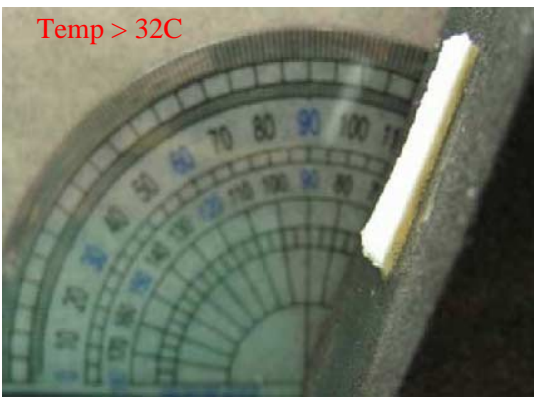

(f)

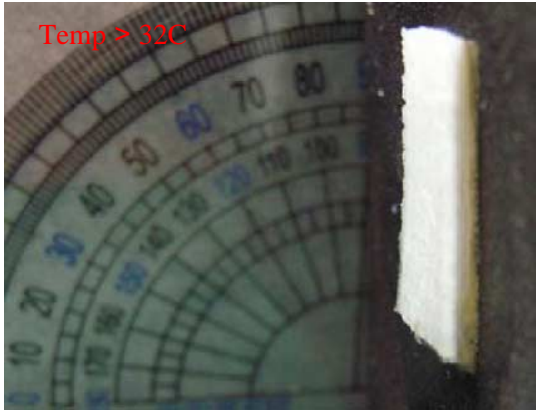

(g)

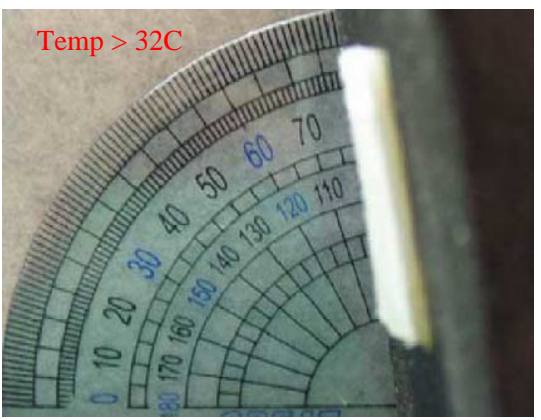

(h)

Fig. 7. Photo of the easily stripped-off trilayer wound dressing. 


\section{Results and discussion}

\subsection{Graft PNIPAAm onto PP nonwoven}

The PNIPAAm hydrogel was grafted onto PP nonwoven by UV-induced modification process. Before grafting PNIPAAm hydrogel onto PP nonwoven, the surface of substrate was modified to a hydrophilic state by Ar plasma pretreatment. From the SEM observation (Fig. 2), the PNIPAAm grafted layer was attached well to the plasma pretreated nonwoven, due to the increase in wettability between hydrogel and substrate. However, there is a large gap between hydrogel and untreated nonwoven, and PNIPAAm was easily to come off from the substrate.

\subsection{Cross-link of chitosan hydrogel}

The results of immobilized chitosan hydrogel onto PP nonwoven were shown in Table 1 . The chitosan could not form a 3-D network structure without the cross-linking agent, glutaradehyde. Moreover, the strength of chitosan hydrogel was actually affected by the concentration of glutaradehyde. The hydrogel was found to change from hard to brittle as the concentration of glutaradehyde increased. The brittleness of hydrogel may limit its bioapplications.

\subsection{Morphological observation}

Fig. 3 shows cross-section structures of chitosan/PP nonwoven composites dried at room temperature and freeze dried at $-80{ }^{\circ} \mathrm{C}$. No any pore was observed inside the chitosan when it was dried at room temperature. On the other hand, porous structure was observed when it was

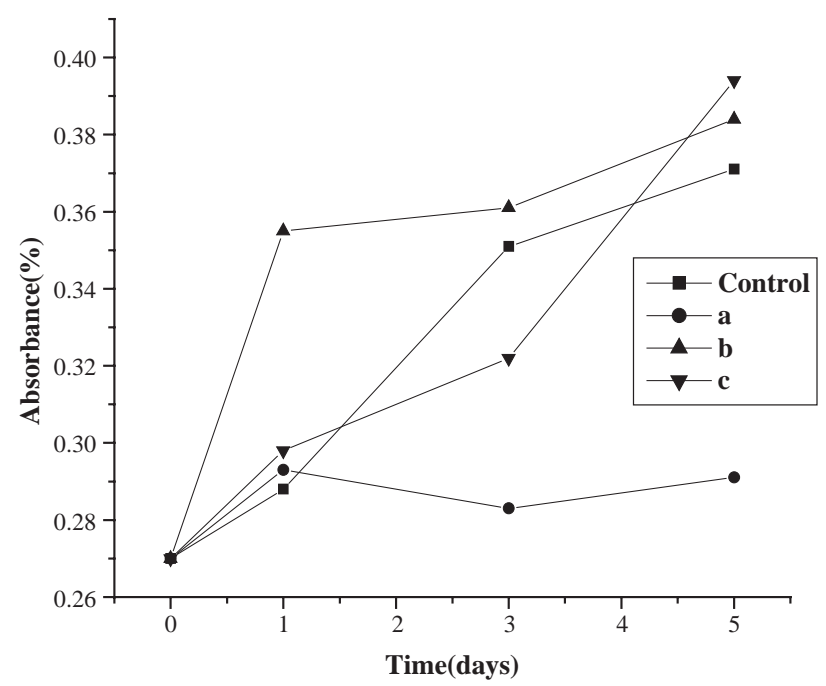

Fig. 8. Cell culture test of (a) untreated PP nonwoven; (b) chitosan; and (c) chitosan immobilized on the PP nonwoven. (evaluated by MTT method).
Table 2

Reduction of E. coli and S. aureus by contact with PP nonwoven and chitosan

\begin{tabular}{lll}
\hline & Reduction rate $(\%)$ & \\
\cline { 2 - 3 } & Escherichia coli & Staphylococcus aureus \\
\hline PP nonwoven & 0 & 0 \\
Chitosan & 85 & 91 \\
\hline
\end{tabular}

Each specimen was immersed in E. coli and $S$. aureus for $2 \mathrm{~h}$ at room temperature. Then the number of bacteria was determined by colony assay.

freeze dried. However, due to this complicated entangle structure between the nonwoven fabric and chitosan hydrogel, the nonwoven was difficult to strip. Consequently, an easy-stripped interface layer was really required for preparing an ideal wound dressing. Therefore, a PNIPAAm hydrogel interface was chosen to solve the entangle problem due to its temperature sensitivity and high hydrophilic property. Chitosan with porous structure was completely covering the PNIPAAm/PP nonwoven composites to form a tri-layer structure (Fig. 4). In this tri-layer, chitosan has an interconnected porous structure and an average pore size of 100 ìm as shown in Fig. 5, freeze-dried process was characterized by the large, open, channel-like appearance, and high porosity structure which is advantageous for cell proliferation.

The main object of this study was to develop an easily stripped-off trilayer wound dressing with an architecture shown in Fig. 6. Normally, the wound dressing is changed once a week. At this moment, due to the biodegradability of chitosan, the chitosan layer degraded eventually and the unbonded PNIPAAm-tissue interface was created. From the Fig. 7(a)-(d), when the wound dressing needs removed, just let the temperature of the substrate below the LCST to make PNIPAAm swelling and then it would be easily stripped-off from the wound surface(when an angle $\theta>70^{\circ}$ ) without damaging the new regenerated tissues [13-16]. On the contrary, from Fig. 7(e)-(h), the temperature of the substrate high the LCST to make PNIPAAm deswelling and then it could not be stripped-off from the wound surface.

\subsection{Cell proliferation}

MTT reagent is a pale yellow substrate which produces a dark blue formazan product when it is incubated with viable cells. Therefore, the level of the reduction of MTT into formazan can reflect the level of cell metabolism. Fig. 8 shows the time course of formazan accumulation for the untreated PP nonwoven, chitosan and chitosan immobilized on the PP nonwoven. The tissue culture polystyrene dish is used as a control group. Formazan absorbance indicates that fibroblasts seeded onto the chitosan and chitosan immobilized on the nonwoven were able to convert the MTT into a blue formazan product. The tendency of these two curves is similar. 


\subsection{Antibacterial activity}

Chitosan was reported to inhibit the growth of bacteria through binding of its positively charged amino groups to negatively charged bacterial cell wall. Therefore, the chitosan hydrogel reduced the number of bacteria achieved by immersing hydrogel into bacteria suspension. The reduction rate of $E$. coli and $S$. aureus number after $2 \mathrm{~h}$ culture contact with a hydrogel is shown in Table 2. As expected, chitosan hydrogel showed $85 \%$ reduction in E. coli number and $91 \%$ reduction in $S$. aureus number, while untreated nonwoven, as a control, did not give any change on bacteria number.

\section{Conclusions}

1. The Argon gas plasma pretreatment could extremely advantageous for processing the photo-induce grafting polymerization to reach the fine graft density and surface hydrophility.

2. NIPAAm could be efficiently grafted onto the surface of PP nonwoven with the additional agents such as APS, TEMED, and NMBA, and a transparent hydrogel layer was formed.

3. The chitosan hydrogel layer can be successfully immobilized onto the surface of PNIPAAm/PP nonwoven composite through cross-linking process. And the porous chitosan sponge structure that suitable for cell growth can also be prepared by subsequently freeze-drying.

4. From fibroblast cell culture by MTT method, the porous chitosan sponge exhibited the good biocompatibility to fibroblast cells.

5. Chitosan hydrogel displayed preliminary antibacterial ability to $E$. coli and $S$. aureus $(85 \%$ and $91 \%$ reduction rate, respectively).

6. This tri-layer wound dressing can be a promise approach for tissue engineering applications.

\section{References}

[1] P. King, Burns 26 (2000) 501.

[2] J. Nanchahal, R. Dover, W.R. Otto, Burns 28 (2002) 254.

[3] H. Yanaga, Y. Udoh, T. Yamauchi, M. Yamamoto, K. Kiyokawa, Y.Y. Tai, Burns 27 (2001) 689.

[4] K. Soejima, M. Nozaki, K. Sasaki, M. Takeuchi, N. Negishi, Burns 23 (1997) 501

[5] Sandra Hall, "Wound dressing", Healthy and Science Television Network subscribers, EDA 211-0033.

[6] B.D. Ratner, A.S. Hoffman, Biomaterials Science-An Introduction to Materials in Medicine, 1996, p. 362.

[7] C. Lorenz, A. Petracic, H.P. Hohl, L. Wessel, K. Waag, Burns 23 (1997) 505.

[8] M.T. Sheu, J.C. Huang, G.C. Yeh, H.O. Ho, Biomaterials 22 (2001) 1713.

[9] H. Kubota, M. Yamamoto, React. Funct. Polym. 29 (1996) 77.

[10] M. Wu, C. Jie, Z. Ding, Z.T. Ma, Radial Phys. Chem. 48 (1996) 525.

[11] L. Liang, X. Feng, L. Peurrung, V. Viswanathan, J. Membr. Sci. 162 (1999) 325

[12] M. Heskins, J.E. Guillet, E.J. James, Macromol. Sci. Chem. A2 (1968) 1441.

[13] K.S. Chen, J.C. Tsai, F.H. Lin, T.M. Chen, T.H. Wu, C.C. Chen, J. Mater. Sci. Eng. 35 (2003) 231.

[14] F.H. Lin, T.M. Chen, K.S. Chen, T.H. Wu, C.C. Chen, Mater. Chem. Phys. 64 (2000) 189.

[15] S.Y. Lin, K.S. Chen, R.U. Liang, Biomaterials 22 (2001) 2999.

[16] C.C. Lin, K.S. Chen, T.H. Wu, C.H. Len, Z.T. Tsai, B. Lin, U.S Patent, 6022330 (2000).

[17] T. Chandy, C.P. Sharma, Artif. Organs 18 (1990) 1.

[18] R.A.A. Muzzarelli, Carbohydr. Polym. 20 (1993) 7.

[19] Y. Shigemasa, S. Minami, Gen. Eng. Rev. 13 (1995) 383.

[20] J.F. Pruden, P. Migel, P. Hanson, L. Friedrich, I. Balassa, Am. J. Surg. 119 (1970) 560.

[21] V.V. Radhankrishnan, M.S. Vijayan, M. Sambasivan, M. Jamaluddin, S.B. Rao, Biomedicine 2 (1991) 3.

[22] K.S. Chen, J.C. Tsai, C.W. Chou, M.R. Yang, J.M. Yang, Mater. Sci. Eng., C, Biomim. Mater., Sens. Syst. 20 (2002) 203.

[23] M.R. Yang, K.S. Chen, Mater. Chem. Phys. 50 (1997) 11.

[24] M.R. Yang, K.S. Chen, J.C. Tsai, C.C. Tseng, S.F. Lin, Mater. Sci. Eng., C, Biomim. Mater., Sens. Syst. 20 (2002) 167.

[25] K.S. Chen, S.F. Lin, Y. Ikada, Chin. J. Med. Biol. Eng. 13 (1993) 211.

[26] T. Mosmann, J. Immunol. Methods 65 (1983) 55. 